possible to see, although with some difficulty, galaxies at this redshift and beyond. Galaxies have two obvious advantages over quasars for our purpose: their space density is much higher, and they do not vary on a timescale shorter than a few billion years. Therefore, if there is a giant gravitational lens then we should be able to see many multiply-lensed galaxies. If these are discovered we shall know there is a lens, and we shall be able to study the lens structure. If the multiply-lensed galaxies are not found then we shall have to conclude that there is no lens. The task is obviously difficult, and it may take years to complete, but I think that any proof may come only from studies of far away galaxies in the field of the 2.6 arc min lens candidate.

\section{P. PACZYŃSKI}

Princeton University Observatory, Peyton Hall, Princeton,

New Jersey 08544, USA

1. Paczyński, P. Nature 319, 567-568 (1986).

\section{'Capture' of stereopsis by illusory contours}

RAMACHANDRAN and Cavanagh ${ }^{1}$ reported an interesting effect in which disparate subjective contours capture regions of zero disparity enclosed within these contours but not regions that lie outside the contours. They suggest that this phenomenon challenges the current computational models of stereo matching. However, the effect is not as general as their article implies: it occurs only in the presence of ambiguity posed by periodic structures. When non-periodic backgrounds are used the stereo capture does not occur (Fig. 1 bottom). This suggests that the phenomenon may have something to do with the treatment of periodic structures by the visual system ${ }^{2}$.

Most current computational models of stereo matching ${ }^{3-5}$ are based on point-bypoint matching of features and use a set of assumptions or constraints (based on some very general properties of the environment) to ease the computational burden associated with solving the often massive local ambiguity problem. Surfaces containing periodic texture cannot be successfully matched locally. For example, a periodic texture on a frontoparallel plane will produce a set of peaks in the crosscorrelation surface, each corresponding to a possible match. Locally, there is not enough information to decide which of these possibilities is the correct one. True correspondences can be obtained only by using more global information or by incorporating information originating outside the stereomatching system. This is true for any matching mechanism relying on local computations.

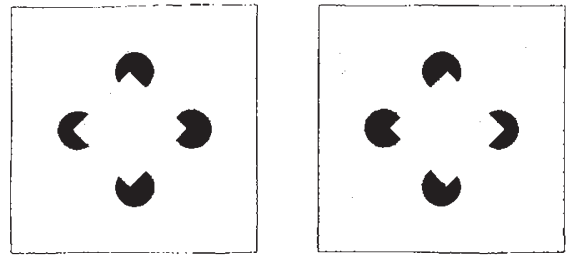

output of retinal receptors is to the perception of brightness.

Rather than challenging current computational models of stereomatching, stereoscopic capture illuminates, I believe, mechanisms underlying more cognitive or configurational aspects of stereoscopic processing.
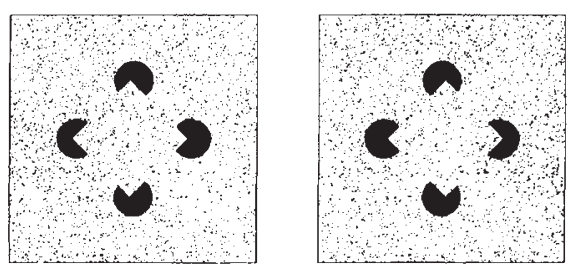

Fig. 1 Depth carried by disparate subjective contours is attributed to periodic (top) but not to non-periodic (bottom) texture elements enclosed by these contours. The stereograms were constructed for viewing with free fusion by crossing the eyes in front of the displays.

The problem of multiple 'local maxima' has been often attacked by invoking multiresolution strategies in which low spatial frequency matches are used to disambiguate the high frequency ones. ${ }^{4}$. However, such strategy is not of much use in the case of extended periodic structures. Another, more general, way to solve this problem is to use other cues, for example, accommodation ${ }^{6}$, to bias disparity. The idea of a set of depth maps cooperating to converge on a state in which all maps agree and present sharp and accurate three-dimensional localization of features is a powerful notion, the potential of which has yet to be fully explored in the machine vision research.

Current computational theories of stereopsis do not address the qualitative or quantitative aspects of binocular depth perception. Rather, they attempt to understand only its first step: the detection of binocular disparities. Not all of them assume that binocular disparities are directly linked to our perception. That is to say, not all of them assume that our perception of depth from stereograms are isomorphic to the state of the neural disparity network. It is possible, to use an analogy, that binocular disparities are to the stereoscopic depth perception what the

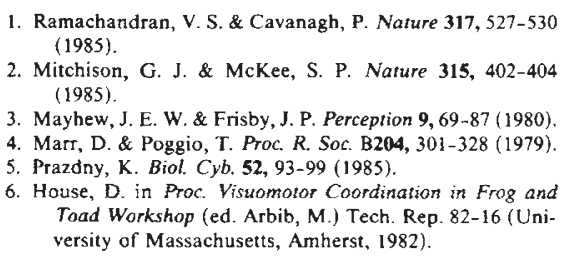

1. Ramachandran, V. S. \& Cavanagh, P. Nature 317, 527-530 (1985).

2. Mitchison, G. J. \& McKee, S. P. Nature 315, 402-404 (1985).

3. Mayhew, J. E. W. \& Frisby, J. P. Perception 9, 69-87 (1980)

4. Marr, D. \& Poggio, T. Proc. R. Soc. B204, 301-328 (1979)

5. Prazdny, K. Biol. Cyb. 52, 93-99 (1985). House, D. in Proc Visuomotor Coordination in Frog and
Toad Workshop (ed. Arbib, M.) Tech. Rep. 82-16 (University of Massachusetts, Amherst, 1982).

\section{K. PRAZDNY}

Machine Perception Group,

Artificial Intelligence Laboratory,

FMC Corporation,

1185 Coleman Avenue,

Box 580, Santa Clara,

California 95052, USA

RAMACHANDRAN REPLIES-in our article on stereoscopic capture ${ }^{1}$ we argued that existing stereo-algorithms could not account for our findings although it is possible that some of them could be modified to do so. This is quite consistent with Prazdny's interesting suggestion. Since disparity of illusory contours can influence the matching of finer elements in a stereogram (Fig. 1, top), we concluded that monocular contours are involved in stereopsis ${ }^{1,2}$. Contrary to Prazdny's suggestion, however, we believe this to be a very general effect and 'capture' happens to be just one way of demonstrating their role. Prazdny also suggests that the effect is specific to 'periodic' patterns, a possibility that we ourselves consider (page 528) when discussing the phase sensitivity of stereo-capture. However, it is almost certainly the lack of ambiguity (not lack of periodicity) that prevents capture in Prazdny's Fig. 1 (lower).

Furthermore, there are also three other aspects of our effect that are not easily handled by existing computational models. First, stereo capture can be seen even in rivalrous displays ${ }^{1,2}$ (especially when flashed briefly) and not just in peri-

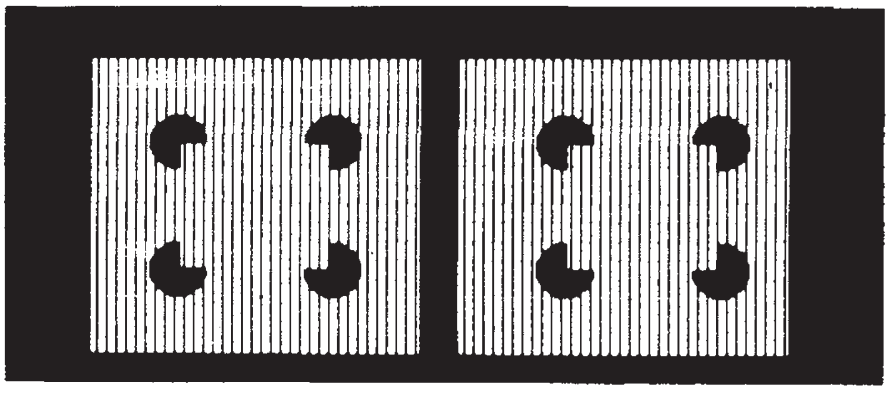

Fig. 2 Stereo capture is not seen if disparity is introduced between the disks themselves. 